\title{
Evidence for an anaphoric mechanism within syntactic processing: Some reference relations defy semantic and pragmatic constraints
}

\author{
WAYNE COWART \\ The Ohio State University, Columbus, Ohio \\ and \\ HELEN S. CAIRNS
}

Queens College, Flushing, New York, and City University of New York, New York

\begin{abstract}
In three experiments, we examined an interaction between the pronoun they and syntactic analysis. Experiment 1 demonstrates that they can slow reading times to is when this verb is visually presented immediately after a sentence fragment ending with an ambiguous expression such as flying kites. This effect seems to involve a coreference assignment linking they and the ambiguous expression that influences the syntactic analysis of the latter. Experiments 2 and 3 show that this effect can operate even when coreference between they and the ambiguous expression is implausible. These results support a modular theory of comprehension that includes structurally oriented reference processes with access to some, but not all, of the listener's knowledge relevant to coreference.
\end{abstract}

The syntactic principles that characterize sentences in natural languages have a distinctive quality that has, as far as we know, no parallel in other domains. Although these principles seem to be quite complex, they are nevertheless employed with such speed and facility as to suggest that their application is a kind of reflex (see Fodor, 1983 , p. iv). This, among many other considerations, has led some investigators to speculate that these principles are brought to bear on sentence comprehension by special cognitive processes dedicated to this task. In particular, the suggestion is that these processes are distinct from and essentially insensitive to those that deploy the interpretive principles and real-world knowledge that, together with the syntactic principles, determine sentence interpretation. For example, the fact that the patient is understood to perform surgery in the sentence The patient consulted the doctor after performing the appendectomy suggests that some process dominated by the structural properties of this sentence overrides any processes that might provide a more plausible analysis based on real-world knowledge. Models of the language comprehension system that stress distinctions among process types and posit

\footnotetext{
This article is based in part on the first author's dissertation, which was done at the City University of New York under the direction of the second author. This project benefited greatly from discussions with Bob Fiengo, Michael Suddert-Kennedy, Terry Langendoen, Lou Gerstman, Merrill Garrett, and Tom Bever. Roberta Klatzky and two anonymous reviewers provided very valuable comments on earlier versions of the manuscript. The authors are grateful to Uma Subramanian for invaluable assistance in executing the third experiment. Requests for reprints should be sent to W. Cowart, Cunz Hall, Room 204, Ohio State University, 1841 Millikin Rd., Columbus, $\mathrm{OH} 43210$.
}

constraints on interactions among types are collectively known as modular or autonomous theories.

The research reported here supports a modular conception of the language comprehension system. We argue that an empirically adequate theory of this system must recognize a distinction between processes that select condidate antecedents for certain pronouns on narrowly structural grounds, and other processes that can use knowledge of selectional restrictions and real-world knowledge to revise or replace antecedent selections made by the first kind of process. Furthermore, although both kinds of process bear directly on the same problem (antecedent selection), it seems to be necessary to posit severe constraints on the extent to which the second kind can influence the operations of the first.

In this article, we describe three experiments. Experiment 1 used a paradigm originated by Tyler and MarslenWilson (1977). It demonstrated that there are circumstances in which a pronoun in prior context can influence subjects' choice of syntactic analysis for a syntactically ambiguous expression. Experiment 2 used the same paradigm with different materials. It demonstrated that this pronoun-based influence cannot be blocked by certain semantic or pragmatic properties that are relevant to the interpretation of the utterance, although this influence apparently is blocked by structural factors. Experiment 3 replicated and extended the results of Experiment 2.

\section{Modularity Theory}

Early formulations of an autonomous comprehension theory were suggested by Forster and his colleagues (Forster, 1976, 1979; Forster \& Olbrei, 1973; Forster \& 
Ryder, 1971). Forster and his collaborators provided numerous demonstrations that processing difficulty can be independently manipulated by structural and plausibility variables. Recently, Frazier and Rayner (1982), Rayner, Carlson, and Frazier (1983), and Ferreira and Clifton (1986) used various reading-based methods to obtain results suggesting that syntactic processes are independent of various semantic and pragmatic considerations. For an extensive review of the modularity literature, see Cairns (1984).

A general formulation of the modularity concept can be found in Fodor (1983). He suggested that a number of cognitive functions (e.g., aspects of linguistic and visual perception) are implemented by domain-specific, automatic cognitive modules (i.e., "input systems") characterized by a number of properties. One such property is that input systems are "informationally encapsulated" (pp. 64-86). For Fodor, this amounts to the claim that only some of the perceiver's knowledge that is actually or potentially relevant to a given input can be exploited by an input system. In particular, he suggested that the linguistic input system is informationally encapsulated in such a way that it cannot use inferences based on realworld knowledge to determine the properties of the representations it produces.

\section{On-Line Interactive Theory}

Marslen-Wilson, Tyler, and their associates have been prominent among the critics of autonomy theory (e.g., Marslen-Wilson, 1973, 1975; Marslen-Wilson \& Tyler, 1975, 1980a, 1980b; Marslen-Wilson, Tyler, \& Seidenberg, 1978; Marslen-Wilson \& Welsh, 1978; Tyler \& Marslen-Wilson, 1982). Their position is that the interaction of semantic and syntactic factors in on-line processing is essentially unconstrained. The studies purporting to demonstrate this, however, have generally been open to alternative interpretations (see Cowart, 1982; Forster, 1979; Townsend \& Bever, 1982).

Perhaps the most persuasive evidence for semantic influences on syntactic processing in this body of work was reported in Tyler and Marslen-Wilson (1977). The principal materials were sentence fragments similar to those in Example 1:

1a. If you walk too near the runway, landing planes...

lb. If you've been trained as a pilot, landing planes...

For the experimental cases, the sentence fragment always ended with an ambiguous expression similar to landing planes (i.e., of the form VERB + ing NOUN $+s$ ). In such cases a subject's choice of an interpretation for the ambiguous expression is related to the form of the following verb. If, in the example, landing planes is interpreted as a plural noun phrase (e.g., Landing planes scare me), then only plural verb forms are appropriate. If landing planes is interpreted as a gerundive nominal (e.g., For untrained pilots to be landing planes scares me), then the appropriate verb form is singular.

The results of Tyler and Marslen-Wilson's (1977) pretest were used to select a pair of sentence fragments for each ambiguous expression, as in Example 1. Each fragment biased subjects toward one of the two interpretations of the ambiguous expression.

On each trial in the experiment that used these materials, subjects heard a sentence fragment and then, immediately afterward, saw either is or are on a tachistoscope. The task was to read the word aloud as rapidly as possible. The interval between the onset of the visual presentation and the subject's response was timed.

Tyler and Marslen-Wilson (1977) compared two cases of particular relevance here. In one the verb form presented after a sentence fragment was compatible with the interpretation of the ambiguous expression toward which that fragment biased the subject. In the other the verb form was not compatible. The results indicated that subjects could name the verb form about $30 \mathrm{msec}$ faster when it was compatible with the bias induced by the fragment.

This finding was interpreted as evidence for an "online, interactive" theory of language processing. In particular, Tyler and Marslen-Wilson (1977) suggested that the semantic interpretation of the subordinate clause ahead of the ambiguous expression had biased the listener's syntactic interpretation of the ambiguous expression itself, perhaps acting to completely suppress the less preferred analysis. This, in turn, influenced the subject's reading of the verb form. Although they acknowledged that other interpretations remained open (see Forster, 1979, for one alternative analysis), Tyler and Marslen-Wilson suggested that these results demonstrated semantic involvement in syntactic processing.

Cowart (1983) and Townsend and Bever (1982) argued that there are several differences between the two classes of fragments used in Tyler and Marslen-Wilson's (1977) study that might have accounted for the results. For present purposes, the most important of these differences involves third-person plural pronouns (e.g., they). Twenty-four of 28 fragments biasing toward are contained they or some closely related form, whereas only one of the fragments biasing toward is contained one of these pronouns. Examples $2 \mathrm{a}$ and $2 \mathrm{~b}$ are representative of many of the fragment pairs Tyler and Marslen-Wilson used:

2a. If you know how to handle sudden gusts of wind, flying kites...

2b. As they glide gracefully over the city, flying kites...

An intuitive analysis of these materials suggests that in Example $2 b$, the fragment that biased toward are, the biasing effect arises largely from the presence of they; they can only be interpreted by reference to some other entity. In an experiment in which sentence fragments such as Example $2 b$ are presented out of context, the only (logical) candidate antecedent for they is flying kites. However, flying kites can play this role only if it is interpreted as a plural noun phrase.

These considerations suggest the possibility that Tyler and Marslen-Wilson's (1977) result was determined 
largely by the asymmetric use of pronouns in the experimental materials. Although the involvement of pronouns suggests a look at the literature on pronominal anaphora, in fact no phenomenon very much like the one reported in the present studies has been previously described. Therefore, a review of existing work is best postponed until the outlines of the phenomenon under consideration here are better defined.

The experimental program outlined below addresses in two phases the theoretical issues arising from Tyler and Marslen-Wilson's (1977) result. The first phase, Experiment 1 , shows that within a sentence a pronoun can influence the syntactic analysis of a following ambiguous expression. The second phase, Experiments 2 and 3, demonstrates that the mechanism responsible for this influence is apparently sensitive to structural limitations, yet oblivious to some of the nonsyntactic information listeners normally use to select pronoun antecedents in discourse contexts. This is, of course, the kind of effect modular theories predict and many interactive theories deny.

\section{EXPERIMENT 1}

Experiment 1 primarily bears on the question of whether naming times to verb targets are sensitive to the presence of they in contexts similar to those of Tyler and Marslen-Wilson (1977). If they are, responses to is could be slowed, responses to are could be accelerated, or both. If there is an interaction involving both verbs, the two should be named about equally rapidly with a lexical noun phrase in context. When they is present, responses to are should be faster than those to is.

\section{Method}

Materials and Design. The materials were modeled on those used by Tyler and Marslen-Wilson and were similar to Example 3:

3a. While the boxes usually come with several internal partitions, packing cases...

3b. While they usually come with several internal partitions, packing cases...

All pairs of items contrasted a lexical noun phrase subject in the subordinate clause, as in Example 3, with the pronoun they. The visual target that followed the fragment was always a form of be. All of the sentence fragments satisfied the following five criteria: (1) each began with a single subordinate clause, followed immediately by a two-word ambiguous expression of the form VERBing NOUNs; (2) the subordinate clauses contained no instances of the verbs be or have in any form (except in one case, an error); (3) none of the subordinate clauses contained any pronoun apart from those specifically manipulated in the experiment; (4) the noun phrase that alternated between they and its control was always the subject of the subordinate clause; and (5) the material intervening between the subject position in the subordinate clause and the ambiguous phrase was of moderate length, from 3 to 15 syllables. The experimental materials are listed in Appendix A.

The design involved two within-subjects factors, context (the subject of the initial subordinate clause was either a lexical noun phrase or they) and verb (is vs. are). There were four subject groups resulting from crossing two materials tapes with two slide sequences.
Procedure. Control of intonation was achieved by retaining the same version of the ambiguous expression on each of two materials tapes. This was always the version recorded in the context of the fragment bearing they. Thus, when complete, the two materials tapes contained phonetically identical versions of the ambiguous expression. Each working tape was an edited copy of a single master tape containing both versions of each item.

There were 80 trials, of which half were fillers. The experiment was run in two blocks of 40 trials each with a 20 -trial practice session at the beginning. The instructions described the sequence of events on a typical trial and told subjects to listen carefully to each sentence fragment and to speak each visually presented word clearly and loudly as soon as it appeared. Subjects were seated before a darkened enclosure, which incorporated the screen of a projection tachistoscope and a microphone. The screen was about $.56 \mathrm{~m}$ from the subject. The subject's field of view was limited to events relevant to the experiment.

Sentence fragments were presented via headphones. At the offset of each fragment, the tachistoscope displayed a word slide and a timer started. The subject's phonation stopped the timer. To ensure that subjects attended to the sentence fragments, they were also asked to indicate, after the initial spoken response, whether the verb presented made a good continuation of the preceding fragment. These responses were not recorded.

Subjects. Twenty-four Queens College undergraduates participated in the experiment. Each was paid $\$ 2$ for his/her services.

\section{Results}

The results are summarized in Figure 1. The graph shows a large difference in naming time to is and are arising when the pronoun they appeared in context. The effect seems to result largely from slowing naming responses to is. When the $i s$ responses are analyzed separately, there is a large context effect $\left[F_{1}(1,20)=\right.$ $17.45, M S \mathrm{e}=902.0, p<.001 ; F_{2}(1,36)=7.79, M S \mathrm{e}$ $=3,518.7, p<.01]$. (Test statistics subscripted 1 are by-subjects analyses and those subscripted 2 are bysentences analyses, as per Clark, 1973.) By contrast, the small speedup in the are responses does not approach significance. In an ANOVA covering both is and are results, the effect of they is reflected in a significant interaction between the context and verb factors $\left[F_{1}(1,20)=13.45\right.$, $M S \mathrm{e}=965.48, p<.002 ; F_{2}(1,36)=6.89, M S \mathrm{e}=$

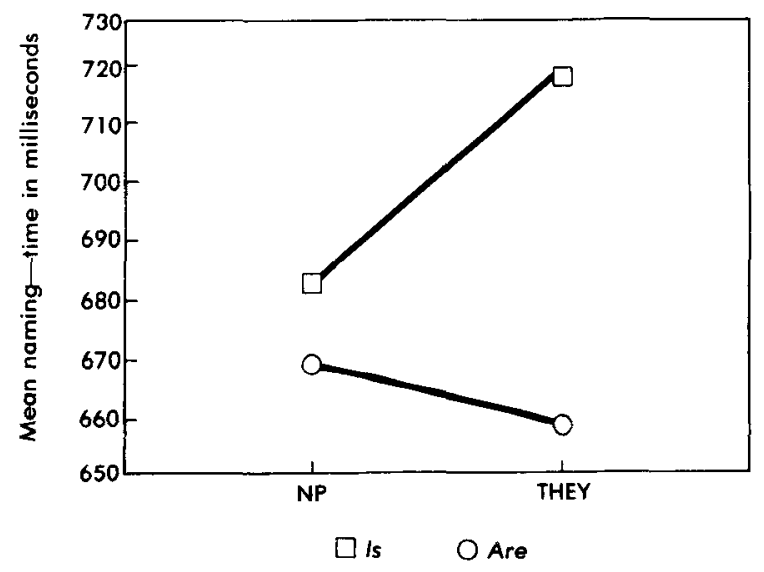

Figure 1. Naming times (in milliseconds) for Experiment 1. NP $=$ noun phrase. 
$3,182.6, p<.02]$. The interaction, however, seems to be almost entirely a reflection of the context effect in the is responses. The slowed responses to $i s$ in the presence of they are also reflected in the main effect of the verb factor $\left[F_{1}(1,20)=9.17, M S e=3,434.8, p<.01\right.$; $\left.F_{2}(1,36)=21.55, M S e=3,031, p<.001\right]$.

There are also two significant but uninteresting interactions with the subject group factor in the by-subjects analysis: group $\times$ verb $\left[F_{1}(3,20)=3.59, M S \mathrm{e}=\right.$ $3,434.8, p<.05]$ and group $\times$ context $\times$ verb $\left[F_{1}(3,20)\right.$ $=6.59, \mathrm{MSe}=965.5, p<.005]$. The latter reflected the fact that responses to are were faster in the presence of they in three groups, whereas in one group responses were slower. For all groups they slowed responses to is.

The by-items analysis included a between-items block factor. Each item was assigned to one of four blocks according to how the various forms of the item were distributed among the materials sets. Three interactions with the block factor were significant: block $\times$ context $\left[F_{2}(3,36)=9.84, M S \mathrm{e}=2,975.6, p<.001\right]$, block $\times$ verb $\left[F_{2}(3,36)=4.71, M S e=3,031.0, p<.01\right]$, and block $\times$ context $\times$ verb $\left[F_{2}(3,36)=32.77, M S \mathrm{e}=\right.$ $3,182.6, p<.001]$. The latter effect is potentially important since it may reflect differing context effects across blocks. However, because the block factor is confounded with differences in mean naming time across subject groups, an analysis was done in which each naming time was replaced by a $Z$ score based on the mean and standard deviation of the group from which it was obtained. In this analysis the second and third interactions listed above were not significant, although the theoretically important effects discussed earlier were largely unchanged. Most important, the effect of they on is is in the same direction for all blocks.

\section{Discussion}

These results support two conclusions. First, the presence of they in prior context can bias the syntactic interpretation of an ambiguous structure such as flying kites. As noted earlier, the apparent source of this effect is that they needs an antecedent for interpretation and that flying kites, or a similar expression, can be that antecedent, provided that it is syntactically construed as a plural noun phrase.

The second conclusion these results support is that the presence of they in context is reflected solely in a slowing of naming time to $i s$ when this verb form is inconsistent with the preferred analysis of the ambiguous expression. The pronoun yields no significant speedup of naming time to are. (This is an important point that will bear on the interpretation of Experiments 2 and 3.) For the purposes of subsequent discussion, we will use the term pronoun bias effect to refer to the slowing of naming responses to is when they appears in ambiguous materials similar to those used here. Note that this definition relates only to is. Responses to are appear to have little or no bearing on the issues at hand.

These findings suggest that the effects observed by Tyler and Marslen-Wilson (1977) resulted from an asymmet- ric distribution of they and related pronouns in the sentence fragments used in that study. As noted above, they was very common among the fragments biasing toward are, but it was used only once in a fragment biasing toward is. Since in Experiment 1 we used the same procedure as did Tyler and Marslen-Wilson (1977), as well as similar materials, and varied only the presence or absence of they, it is clear that this pronoun alone can produce the observed effect. Note that the is/are difference with the pronoun in context is much larger than the comparable difference observed by Tyler and Marslen-Wilson.

These observations are not necessarily damaging to the analysis Tyler and Marslen-Wilson proposed for their findings. The effect appears to involve a coreference relation, which suggests that interpretive processing of the subordinate clause is indeed exerting a "downward" influence on the resolution of the syntactic ambiguity.

There is, however, at least one aspect of Tyler and Marslen-Wilson's proposed interpretation that bears closer examination. Their analysis clearly predicted that all of the semantic and interpretive factors that bear on decisions about anaphoric relations should be involved in the process that relates they to the ambiguous expression in Experiment 1. This implication is made explicit in Marslen-Wilson and Tyler (1980b).

\section{EXPERIMENT 2}

The issue can now be framed as follows: do the procedures that link they to the following ambiguous expression assess both the structural and interpretive aspects of that relation? If the pronoun bias effect can be blocked or attenuated when some interpretive property of the input makes coreference implausible, then this would support the on-line interactive view. If, however, the pronoun bias effect seems to operate even when interpretive aspects of this input lead to an anomalous interpretation, then this would suggest that the device(s) that implement the pronoun bias effect are able to exploit only some of the subject's knowledge relevant to reference relations. Modular theory suggests this possibility.

The aim of Experiment 2 was to determine whether three kinds of information relevant to the interpretation of pronouns such as they are equally involved in the pronoun bias effect. The sentence fragments that follow exemplify the three information types:

4a. If they want to save money, visiting uncles...

$4 \mathrm{~b}$. If they want to believe that visiting uncles...

5a. Even though they use very little oil, frying eggs...

5 b. Even though they eat very little oil, frying eggs...

6a. Whenever they smile during the procedure, charming babies...

$6 \mathrm{~b}$. Whenever they lecture during the procedure, charming babies...

The " $a$ " examples are essentially similar to the materials used in Experiment 1 . In each of the " $b$ " examples, however, an anomalous interpretation results if the they 
is taken as coreferential with the ambiguous expression at the end. These interpretations are anomalous for different reasons. In Example $4 b$ the anomaly seems attributable to structurally based limitations on coreference (see Chomsky, 1981, pp. 183-222; van Riemsdijk \& Williams, 1986, chap. 17). In Example 5b the relevant principle is a selection restriction on eat; specifically, this verb requires an animate subject. Thus, it appears that they is taken as animate when it occurs in this context. On some accounts selection restrictions are syntactic phenomena (e.g., Chomsky, 1965) and on others they are semantic (e.g., Jackendoff, 1972; McCawley, 1968a, 1968b). In Example $6 \mathrm{~b}$ it appears that pragmatic knowledge about what kinds of beings can lecture is used to assign some property of adultness or maturity to the referent of they.

Tyler and Marslen-Wilson's (1977) analysis of their findings clearly suggests that structural, pragmatic, and selectional information will all block coreference. Modular theories in the spirit of Forster (1979) or Fodor (1983) allow the possibility that structural factors, but not pragmatic factors, will block the pronoun bias effect. In a modular theory, the selectional cases might be expected to fall with either of the other two, depending upon whether they are properly viewed as syntactic or semantic in character.

More specifically, both interactive theory and modular theory predict that they should slow responses to is with all the "a" versions of Examples 4-6. Interactive theory predicts this effect will be suppressed by all of the " $b$ " versions of Examples 4-6; that is, anomalies based on structural, selectional, or pragmatic knowledge should all block the pronoun bias effect. The general framework of modular theory suggests the possibility that the pronoun bias effect is implemented by some mechanism that has access only to structural information. In this account, the slowing of responses to is should appear, despite pragmatic anomalies, and should be suppressed by structural anomalies. If this prediction is supported, then selectional anomalies will suppress the pronoun effect only if selectional information is available to the mechanism that implements the pronoun bias effect. The critical differences between interactive and modular theories are that the former predicts that all anomalies will suppress the pronoun effect whereas the latter predicts that pragmatic information, and possibly selectional information, will not block the effect.

\section{Method}

Materials and Design. Twenty-four examples represented each of the three information types illustrated in Examples 4-6. Each fragment was used in eight versions. One dimension of variation was anomaly: each of the " $b$ " versions of Examples 4-6 is considered anomalous. (Note, however, that anomaly arises only where they is taken to be coreferential with the ambiguous expression at the end.) Paired with each anomalous version was a non-anomalous version, as in the " $a$ " examples. Within each of the six cells that result from crossing the three information types with the two anomaly conditions, there was essentially a replication of the design of Experiment 1 (with different control cases). That is, both the anomalous and non-anomalous cases in Examples 4-6 appeared with both a they and a you subject. The four recorded versions of each fragment resulting from these two contrasts were each followed equally often by visual presentations of $i$ and are. The eight versions of each item are illustrated in Example 7, based on Example 4:

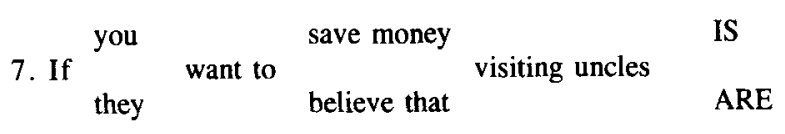

Each sentence fragment was required to satisfy a number of criteria intended to ensure, among other things, that all versions of each fragment were equally plausible. The more important criteria are summarized in Appendix B. The complete list of experimental materials for Experiment 2 is in Appendix C.

Mixed with the experimental fragments were 72 fillers of three types. One third included ambiguous VERBing NOUNs expressions, but these fragments were not interrupted until one or more words after this expression. In another third the fragments were interrupted immediately after an ambiguous VERBing NOUNs expression, but for these the visually presented verb form was never a form of $b e$. Finally, one third of the fragments used non-ambiguous VERBing NOUNs expressions, half of which were compatible with is and half with are.

The design involved three within-subjects factors: anomaly type (structural, selectional, pragmatic), anomaly (non-anomalous, anomalous), and context ( $y o u$ vs. they as the subject of the initial subordinate clause). This design was replicated with each of two target verbs, is and are; the results for the two verbs will be treated separately. A between-subjects groups factor reflected to which of eight materials conditions each subject was exposed. Each subject was represented in the principal by-subjects analysis for each verb by 12 naming-time values, one for each cell of the anomaly type $x$ anomaly $x$ context interaction. Each of these 24 values was the mean of three naming times.

Subjects and Procedure. The analyses below are based on data from 32 Queens College undergraduates. Four additional subjects participated but were excluded from the analysis because sufficient additional subjects to balance the eight materials groups were not available. The four excluded subjects were those having the highest number of missing scores in their respective materials groups. Each subject was paid $\$ 4$.

The preparation of tapes and other materials was as described for Experiment 1. The procedure was likewise unchanged, except that each session was divided into four blocks of 36 trials each, with a short rest between blocks.

\section{Results}

The results are summarized in Table 1, which covers responses to both is and are targets. As suggested by the previous experiment, none of the theoretically important

Table 1

Overall Naming Time (in Milliseconds) for Experiment 2

\begin{tabular}{lrrc}
\hline & \multicolumn{2}{c}{ Context } & Context \\
\cline { 2 - 3 } Verb & $y o u$ & they & \\
Effect*
\end{tabular}

Note-The values in the table were obtained by collapsing across levels of the anomaly type and anomaly factors. $*$ These values were obtained by subtracting each value in the you column from the corresponding value in the they column. TEach value in this row is the difference between is and are values for the column. 
main effects or interactions was significant in an analysis confined to the are results. All further discussion will be concerned only with responses to is.

The pronoun bias effect was replicated across the experiment as a whole; responses to is slowed from 582 to $610 \mathrm{msec}$ when they replaced you in context. This resulted in a significant main effect of context $\left[F_{1}(1,24)=6.99\right.$, $M S e=11,275, p<.02]$.

The most important result, however, is that the pronoun bias effect persists in the face of selectional and pragmatic anomalies. Note that in Figure 2 there is a substantial slowing of responses to is in the selectional and pragmatic anomalous conditions. As expected, structural anomalies do seem to block the effect; in the structural anomalous condition there is no slowing of responses to $i s$. The critical interaction, anomaly type $\times$ anomaly $x$ context, was significant $\left[F_{1}(2,48)=3.45, M S \mathrm{e}=3,411.1, p<.05\right]$. Evidently, the coreference relation underlying the pronoun bias effect is formed even when it leads to an odd interpretation, but it is blocked when it would violate a structural constraint.

A complete set of means for this experiment is shown in Table 2. A priori tests (one-tail) on the slowing of responses to is with they in context showed that this effect is significant in the anomalous condition only on the selection (559 vs. $621 \mathrm{msec}\left[t_{1}(31)=3.37, S E=18.4\right.$, $p<.01]$ and pragmatic (573 vs. $622 \mathrm{msec})\left[t_{1}(31)=\right.$ 3.47, $S E=14.3, p<.001]$ levels of the anomaly type factor. As predicted, the effect does not approach significance in the structural anomalous case (610 vs. $611 \mathrm{msec})$. In the non-anomalous condition the context effect attains or approaches significance only in the structural $(587$ vs. $615 \mathrm{msec})\left[t_{1}(31)=1.92, S E=14.4\right.$, $p<.05]$ and selection cases ( 570 vs. $593 \mathrm{msec})\left[t_{1}(31)\right.$ $=1.5, S E=15.2, p<.1]$. The pronoun bias effect was clearly not replicated in the pragmatic non-anomalous condition (592 vs. $600 \mathrm{msec}$ ).

Although neither is of any interest, there were two other significant effects. There was a main effect of anomaly type $\left[F_{1}(2,48)=4.91, M S \mathrm{e}=2,616.7, p<.02\right]$ and

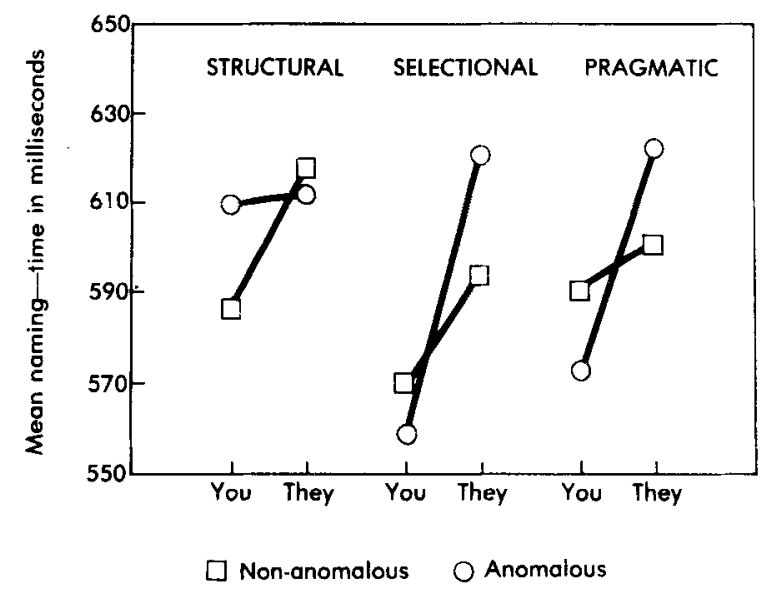

Figure 2. Naming times (in milliseconds) by anomaly type for is targets, Experiment 2.
Table 2

Context Effects on Naming Time to Is (in Milliseconds) for Experiment 1

\begin{tabular}{lccc}
\hline & \multicolumn{2}{c}{ Context } & Context \\
\cline { 2 - 3 } Anomaly Type* & you & they & \\
Effect $\dagger$ \\
\hline Anomalous \\
Structural (4b) & 610 & 611 & 1 \\
Selectional (5b) & 559 & 621 & 62 \\
Pragmatic (6b) & 573 & 622 & 49 \\
& & \\
& Non-anomalous & \\
Structural (4a) & 587 & 615 & 28 \\
Selectional (5a) & 570 & 593 & 23 \\
Pragmatic (6a) & 592 & 600 & 8 \\
\hline
\end{tabular}

Note-A large positive context effect value is indicative of the pronoun bias effect. *The labels in parentheses refer to example sentence fragments 4-6 discussed in the introduction to Experiment 2. †These values were obtained by subtracting values in the they column from corresponding values in the you column.

an interaction among the groups, anomaly type, and anomaly factors $\left[F_{1}(14,48)=2.33, M S \mathrm{e}=3,005.2\right.$, $p<.02]$.

Turning to the by-sentences analysis, the results were much weaker; only the main effect of context $\left[F_{2}(1,69)\right.$ $=4.45, M S e=11,845, p<.05]$ and the context effect within the pragmatic anomalous condition $\left[t_{2}(23)=1.8\right.$, $S E=27.8, p<.05$ (one-tail)] were significant. This raises the question of whether the results might be dependent upon the particular materials used.

In overview, these results provide a general replication of the pronoun bias effect and show no evidence that the effect is reduced when the coreference relation is semantically or pragmatically odd.

\section{Discussion}

The most important result of Experiment 2 is that selectional and pragmatic impediments did not block the pronoun bias effect. It appears that some process makes a preliminary coreference assignment between they and the VERBing NOUNs expression where there is no structural impediment and that the listener's knowledge of the selectional and pragmatic properties of these materials is irrelevant to this process.

There was no evidence of differences between selectional and pragmatic anomalies. Assuming that the syntactic processing system was responsible for blocking the pronoun bias effect where structural anomalies were present (see General Discussion), this suggests that the syntactic system does not deal with selectional restrictions.

Since responses to $i s$ are apparently unaffected by the presence of they in the structural anomalous condition, it seems likely that syntactic relations that block coreference between they and the VERBing NOUNs expression also block the pronoun bias effect. Note, however, that there is an alternative explanation. Although the reasons are not at all clear, naming time to is with you in subject position is far longer in the structural condition than in the selection and pragmatic conditions. Thus there may have been no increase in naming time to $i s$ with they sub- 
jects in the structural condition merely because the values obtained with you subjects were already elevated. Although it seems extremely unlikely that the pronoun bias effect overrides the syntactic impediments appearing in the structural condition, an unambiguous demonstration of syntactic effects on the pronoun bias effect must await further experimentation.

\section{EXPERIMENT 3}

Experiment 3, a partial replication of Experiment 2, was intended to provide further evidence on the effects discussed above and to overcome two limitations of the earlier procedure. As noted above, at the end of each trial subjects made a yes/no decision as to whether the target word made a "good" continuation of the fragment they had heard. The earlier procedure did not provide reliable records of these judgment responses or of any disfluencies or errors subjects produced in speaking the target word.

The predictions for the judgment results parallel those for the corresponding conditions of Experiment 2; in conditions where naming responses to is were predicted to slow, the frequency of "no" judgments should increase. The naming predictions are unchanged.

\section{Method}

Materials and Design. The materials used were drawn from those developed for Experiment 2, apart from minor amendments. The fillers used were also derived from Experiment 2.

The replication eliminated the non-anomalous condition and the selection cases. Thus the experiment was a $2 \times 2$ design involving anomaly type (structural vs. pragmatic) and context (you vs. they). There was also a between-subjects groups factor based on the four materials sets. This design was used with is and are targets, which will be treated separately.

Subjects and Procedure. Sixteen Ohio State University undergraduates participated in the experiment and each was paid $\$ 3.50$.

The outline of the procedure was very much like Experiment 2 except that it was implemented on a computer, which recorded both

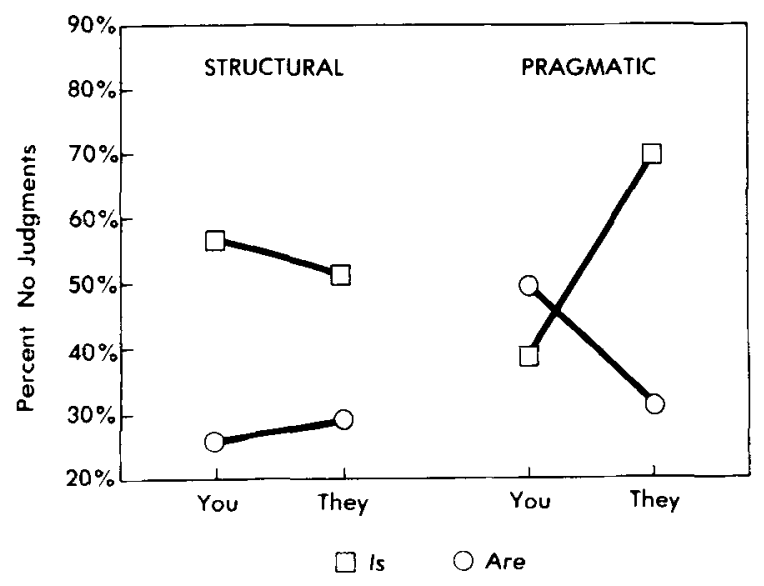

Figure 3. Percentage of "no" responses to the question whether the target verb made a good continuation of the sentence fragment, Experiment 3. (The "no" responses are used to facilitate comparison to naming-time results.)
Table 3

Judgment Data for Experiment 3

\begin{tabular}{|c|c|c|c|}
\hline \multirow[b]{2}{*}{ Anomaly Type } & \multicolumn{2}{|c|}{ Context } & \multirow{2}{*}{$\begin{array}{l}\text { Context } \\
\text { Effect* }\end{array}$} \\
\hline & you & they & \\
\hline \multicolumn{4}{|c|}{ Target: $I s$} \\
\hline Structural & $57 \%$ & $51 \%$ & $-6 \%$ \\
\hline Pragmatic & $38 \%$ & $70 \%$ & $32 \%$ \\
\hline \multicolumn{4}{|c|}{ Target: Are } \\
\hline Structural & $26 \%$ & $27 \%$ & $1 \%$ \\
\hline Pragmatic & $49 \%$ & $31 \%$ & $-18 \%$ \\
\hline
\end{tabular}

Note--The value in each cell shows the percentage of "no" responses obtained in that condition. A "no" response indicates that the subject did not find the target verb a suitable continuation of the preceding sentence fragment. Where the target verb was is, a large positive value in the context effect column is indicative of the pronoun bias effect. *These values were obtained by subtracting values in the you column from corresponding values in the they column.

the subject's naming time and the judgment response made at the end of each trial. The subject was also given a feedback message at the end of each trial showing response time. Subjects were instructed to monitor this message and to try to keep the responsetime values as low as possible. The experimenter monitored the subject's spoken response and recorded any disfluencies or word substitutions that appeared.

\section{Results}

The results of Experiment 3 are summarized in Figures 3 and 4 and Tables 3 and 4 . The judgment data show a clear replication of the pronoun bias effect in the face of anomalous coreference relations, as well as an effect on responses where are is the target. The naming data also show the pronoun bias effect despite anomalous coreference.

Judgment data. In judgments where is was the target, there was a reliable anomaly type $\times$ context interaction $\left\{F_{1}(1,12)=117.34, M S e=.1823, p<.001 ; F_{2}(1,46)\right.$ $=19.45, \mathrm{MSe}=.7332, p<.001]$. This indicates that the increased frequency of "no" responses in the presence of they in the pragmatic condition is reliably different from the pattern seen with structural anomalies. In other terms, a context effect on judgments parallel to the earlier naming effects was observed in the presence of pragmatically anomalous coreference relations. This conclusion is further supported by an analysis covering only the pragmatic condition; this showed a reliable context effect $\left[F_{1}(1,12)=\right.$ 43.03, MSe $=.6979, p<.001 ; F_{2}(1,23)=31.08, M S e$ $=.6295, p<.001]$. The context effect in this condition dominated the overall context effect in the is data, which was also significant $\left[F_{1}(1,12)=10.03, M S \mathrm{e}=.9740\right.$, $\left.p<.01 ; F_{2}(1,46)=8.88, M S \mathrm{e}=.7332, p<.005\right]$. In the by-subjects analysis there was also a significant group $\times$ anomaly type $\times$ context interaction $\left[F_{1}(3,12)\right.$ $=11.29, M S \mathrm{e}=.1823, p<.001]$. Examination of the patterns in various groups reveals that the direction of the context effect is uniform for all groups in the pragmatic condition but varies in the structural condition.

The judgment results where are was the target showed trends opposite those with $i s$. Note that where the coreference relation biases subjects toward the plural noun phrase 
reading of the ambiguous expression, the plural verb form, are, should be more acceptable as the target.

This is what happened in the pragmatic condition. In particular, they in context made are targets more acceptable (reduced "no" judgments), even though the coreference relation was pragmatically odd. (Recall that only the anomalous versions of the materials of Experiment 2 were used here.) The contrasting patterns on the structural and pragmatic levels of the anomaly type factor were reflected in a significant interaction between anomaly type and context (you vs. they) $\left[F_{1}(1,12)=10.34, M S e=.4896\right.$, $\left.p<.01 ; F_{2}(1,46)=5.36, M S \mathrm{e}=.6295, p<.05\right]$. The context effect was also significant in an analysis confined to the pragmatic condition $\left[F_{1}(1,12)=11.56, M S \mathrm{e}=\right.$ $.7813, p<.01 ; F_{2}(1,23)=7.92, M S \mathrm{e}=.7600$, $p<.01]$. The results within the pragmatic condition were also reflected in a main effect of context across all the are data $\left[F_{1}(1,12)=6.51, M S e=.6146, p<.05\right.$; $\left.F_{2}(1,46)=4.24, M S e=.6295, p<.05\right]$. There was also a main effect of anomaly type in the by-subjects data $\left[F_{1}(1,12)=15.13, M S \mathrm{e}=.6979, p<.005\right]$.

Naming-time data. As in Experiment 2, the analysis of the are naming-time data revealed no significant main effects or interactions. This analysis will, as before, be limited to the results where is was the target.

Although the contrast betweeen the naming-time results with is in the two anomaly type conditions appears to be dramatic in Figure 4, the anomaly type $\times$ context interaction was not significant $\left[F_{1}(1,12)=3.91\right.$, n.s.; $F_{2}(1,46)$ $=2.06$, n.s.]. The context effect, however, was reliable when the analysis was confined to the pragmatic condition $\left[F_{1}(1,12)=8.94, M S e=696.23, p<.02 ; F_{2}(1,23)\right.$ $=4.88, M S e=2162, p<.05]$. The subjects analysis also showed a significant main effect of anomaly type $\left[F_{1}(1,12)=5.75, M S e=887, p<.05\right]$.

Despite the fact that responses in the naming task were quite rapid, errors were too rare to permit meaningful analysis, occurring on about $0.5 \%$ of the experimental trials.

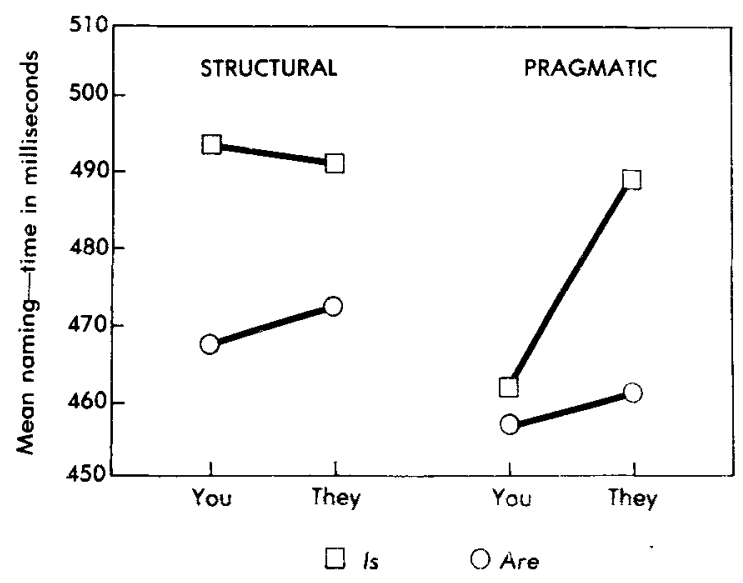

Figure 4. Naming times (in milliseconds) for Experiment 3.
Table 4

Naming-Time Data (in Milliseconds) for Experiment 3

\begin{tabular}{lccc}
\hline & \multicolumn{2}{c}{ Context } & Context \\
\cline { 3 - 4 } Anomaly Type & you & they & \\
Effect*
\end{tabular}

*These values were obtained by subtracting the values in the you column from corresponding values in the they column.

\section{Discussion}

These results reaffirm the most important result of Experiment 2, the evidence that they can exert an influence on the syntactic interpretation of a VERBing NOUNs expression even when a coreference relation between these expressions is implausible.

The judgment effects are much more robust than any of the naming results. There is, however, an interesting contrast between the judgment results of Experiment 3 and the naming-time results of Experiments 1 through 3. None of the naming-time analyses show that they, relative to you, significantly facilitates naming responses to are. However, they in context does increase the judged acceptability of are, although this effect is relatively small.

These results also show that the materials used in the structural conditions in both Experiments 2 and 3 did not function as intended. Subjects find is relatively unacceptable even with you in context. Thus, there appears to be little latitude for the introduction of they to make this target form less acceptable.

Although the results of Experiment 3 considerably strengthen those of Experiment 2, they do not rule out the possibility that they are dependent upon peculiarities of the procedures or materials used here. Subsequent studies (Cowart, 1986a, 1986b), although preliminary, suggest that the pronoun bias effect occurs in further contexts and is demonstrable with quite different experimental procedures.

\section{GENERAL DISCUSSION}

In overview, the principal results of the experiments reported above support two theoretically important conclusions. First, there are circumstances in which the presence of a pronoun in prior context can influence the syntactic analysis assigned to a following structurally ambiguous constituent. This results in the pronoun bias effect observed in the series of experiments reported here. Second, even though a coreference relation seems to be central to the pronoun bias effect, it is not sensitive to selectional or pragmatic influences that are otherwise commonly involved in matters of reference. Specifically, the coreference relation is assigned even when the resulting interpretation is bizarre. 
The most straightforward account of these results seems to be one that distinguishes between two kinds of reference-related mechanisms, one class that has access to interpretive information and another that does not. This approach is developed in more detail below.

The results reported here are incompatible with interactive theories of the sort proposed by Tyler and MarslenWilson (1977) and Marslen-Wilson and Tyler (1980a, $1980 \mathrm{~b})$. Such theories predict that semantic and pragmatic influences on the selection of antecedents will be more or less integral with structural influences.

Although Forster's (1979) modular account of syntactic processing did not directly address the issue of syntactically determined pronominal reference, the results of these studies can be easily accommodated within the general framework of his theory. What is required is simply that the processes that assign the coreference relation underlying the pronoun bias effect be viewed as an extension to Forster's syntactic processor. The relevant effects can then be obtained by further assuming that the syntactic processor can resolve syntactic ambiguities as long as the information used to guide the decision is itself structural. This interpretation is compatible with Forster's general view, because his model incorporates what Fodor (1983) would term an "informationally encapsulated" syntactic processing mechanism; the mechanism is assumed to have access only to a body of specifically structural information and not to much other information that is otherwise relevant to the analysis and interpretation of utterances.

A more detailed account of the results reported here can be built around two central claims: (1) there are two kinds of processes involved in comprehending reference relations, structurally oriented processes and interpretively oriented processes, and (2) information can flow from structurally oriented processes to interpretively oriented processes, but not vice versa. Note that this claim is logical, not temporal; it says nothing about when each process, relative to the other, begins to work on incoming material.

The essential content of the model resides in the distinction between structurally oriented and interpretively oriented processes. Unfortunately, the existing literature, together with the present work, imposes only weak constraints on any such distinction. Nevertheless, there seem to be three dimensions along which it may be useful to distinguish these processes: (1) the kind of representation of the incoming utterance over which the processes are defined, (2) the means by which pronouns are differentiated from one another, and (3) the character of the relations the processes establish. Structural reference processes can be defined over a representation that captures the kinds of properties and relations expressed by some form of phrase marker in linguistic theory. That is, they require representations that specify which lexical items are involved and what their grammatical categories are, as well as how the lexical constituents are assembled into phrasal constituents and what nesting relations there may be among phrasal constituents. Various relations are definable over such structures.

Many processing studies suggest mechanisms that treat pronouns differently according to the context, often the semantic context, in which each occurs. This suggests the second respect in which structurally and interpretively oriented reference processes might be distinguished. The pronoun effects reported here could be implemented by a process that ignores context and differentiates pronouns only according to morphologically realized parameters such as case, gender, number, and so forth.

Finally, McKoon and Ratcliff (1980) and Dell, McKoon, and Ratcliff (1983) found evidence that anaphoric expressions activate not only their antecedents but also other material in the same clause as the antecedent. This suggests a high degree of integration; information derived from one constituent seems to be extended via semantic and discourse relations to others that are interpretively linked to the first. Such power is not needed to implement the pronoun bias effect. For these purposes, it is sufficient if the structural reference processes merely add a pointer or index to the representation of the anaphor indicating which constituent serves as its antecedent.

Thus, there seem to be three ways in which the minimal resources required to implement the pronoun bias effect differ from those suggested by previous work on anaphoric processes. An adequate mechanism could (1) operate on syntactic representations, (2) recognize only lexically, morphologically, or structurally explicit distinctions, and (3) link, but not integrate, coreferential expressions.

The proposed structural reference processes are essentially syntactic. The real-time procedures the model implies are ones in which structural reference processes select candidate antecedents for certain pronouns. The syntactic system chooses among alternative syntactic analyses when it can do so according to criteria defined over syntactic representations. It is directly influenced by coreference assignments made by structural reference processes because they inhabit essentially the same representational domain as do general syntactic processes. The suggested reference relations are passed to the discourse processes, and these processes evaluate them (and sometimes alter them) in the light of the much richer information available in their domain.

Although syntactic representations provide enough information to implement the pronoun bias effect, they seem unable to support many of the anaphoric phenomena revealed by previous research. The general thrust of many experimental studies of reference processing has been that listeners rely upon their general knowledge of the world, among other things, to select pronoun antecedents. Among the particular factors suggested are "implicit causality" (Caramazza, Grober, Garvey, \& Yates, 1977; Ehrlich, 1980; Garvey \& Caramazza, 1974; Garvey, Caramazza, \& Yates, 1974), relative plausibility of alternative coreference assignments (Hirst \& Brill, 1980), and "semantic distance"' (Garrod \& Sanford, 1977). A somewhat more 
explicit proposed influence is the parallel function strategy suggested by Grober, Beardsley, and Caramazza (1978). Marslen-Wilson and Tyler (1980b) provided evidence of very rapid use of plausibility in naming pronouns presented in sentence context. Evidence cited by Clark and Marshall (1981) suggests that very complex reasoning processes exploiting knowledge about the domain of discourse and about the speaker are involved in the analysis of definite reference. Murphy (1984) argued specifically that syntactic representations are too impoverished to account for the phenomena he described, and that something like a discourse model must be invoked.

By contrast, Corbett and Chang (1983) reported on a series of experiments using a probe-latency paradigm that seems to lead toward a model bearing a family resemblance to the one proposed here. They argued that their results support a multiple-access theory of antecedent selection in which the subject is assumed to access all potential antecedents of a pronoun, regardless of the context in which the pronoun occurs. This result seems to favor a two-stage theory of anaphora resolution of the sort that might be suggested by modular theories of language comprehension. Matthews (1986) demonstrated a purely syntactic variable, depth of embedding, affecting preference for coreference.

The work reported here is also related to that of Townsend and Bever (1982). They, too, advocated a differentiated account of the language comprehension system, but one that stresses limitations on interactions that arise from the kinds of representations the system computes. Their research also addressed the claims of Tyler and MarslenWilson (1977), but they manipulated other aspects of the materials. They found that explicit morphological marking of the number property of the initial subordinate clause and the semantic character of the conjunction introducing that clause, among other things, were relevant to the subject's selection of an interpretation of the VERBing NOUNs expression. The effects demonstrated in the present study were achieved despite our having used the widest possible variety of subordinating conjunctions. The morphological bias Townsend and Bever detected also cannot explain the results obtained here; in all cases, the control for they was morphologically and syntactically plural.

The account of the structural reference processes outlined above leads to many further questions. For example, it seems reasonable to expect a noun phrase lying across a sentence boundary to the left of they to eliminate the pronoun effect if it is taken as the antecedent of the pronoun. However, the proposed syntactic account of the pronoun effect suggests that antecedents ahead of the current sentence will be irrelevant to the pronoun bias effect on the assumption that the maximal domain of a syntactic process is a single sentence. (See Cowart, 1986a, for a preliminary report on relevant research.) Other questions concern the relevance of the structural reference processes to singular, third-person pronouns such as he, $s h e$, and $i t$, as well as the role of the processes underlying the pronoun bias effect in the typical case in which the antecedent precedes the pronoun.

To conclude, the work reported here demonstrates that there are circumstances in which the presence of the pronoun they can exert an influence on the syntactic analysis of a following ambiguous expression. The mechanism involved seems to respect syntactic constraints on coreference, but not semantic or pragmatic constraints. Thus it seems that this effect is a manifestation of a reference-assigning mechanism that (1) operates within the syntactic processing system and (2) exploits only the kinds of information present there.

\section{REFERENCES}

CAIRNS, H. S. (1984). Research in language comprehension. In R. Naremore (Ed.), Language science (pp. 211-242). San Diego: College Hill Press.

Caramazza, A., Gorber, E., Garvey, C., \& Yates, J. (1977). Comprehension of anaphoric pronouns. Journal of Verbal Leaming \& Verbal Behavior, 16, 601-609.

CномsкY, N. (1965). Aspects of the theory of syntax. Cambridge: MIT Press.

CHOMSKY, N. (1981). Lectures on government and binding. Dordrecht, The Netherlands: Foris Publications.

Clark, H. H. (1973). The language-as-fixed-effect fallacy: A critique of language statistics in psychological research. Journal of Verbal Learning \& Verbal Behavior, 12, 335-359.

Clark, H. H., \& Marshall, C. (1981). Definite reference and mutual knowledge. In A. Joshi, B. Webber, \& I. Sag (Eds.), Elements of discourse understanding (pp. 10-63). Cambridge, England: Cambridge University Press.

Corbett, A. T., \& Chang, F. R. (1983). Pronoun disambiguation: Accessing potential antecedents. Memory \& Cognition, 11, 283-294.

CoWART, W. (1982). Autonomy and interaction in the language processing system: A reply to Marslen-Wilson \& Tyler. Cognition, 12 , 109-117.

COWART, W. (1983). Reference relations and syntactic processing: Evidence of a pronoun's influence on a syntactic decision that affects word naming. Doctoral dissertation, City University of New York. Available from Indiana University Linguistics Club, Bloomington, IN.

CoWART, W. (1986a). Evidence for a strictly sentence-intemal antecedent-finding mechanism. In M. van Clay, M. Niepokuj, \& V. Nikiforidou (Eds.), Proceedings of the 12th Annual Meeting of the Berkeley Linguistics Society (pp. 41-50). Berkeley: Berkeley Linguistics Society.

CowarT, W. (1986b). Evidence for structural reference processes. In A. Farley, P. Farley, \& K.-E. McCullough (Eds.), Proceedings of the 22nd Regional Meeting of the Chicago Linguistics Society (pp. 307317). Chicago: Chicago Linguistics Society.

Dell, G. S., McKoon, G., \& RATClifF, R. (1983). The activation of antecedent information during the processing of anaphoric reference in reading. Journal of Verbal Learning \& Verbal Behavior, 22 , 121-132.

EHrLICH, F. (1980). Comprehension of pronouns. Quarterly Journal of Experimental Psychology, 32, 247-255.

Ferreira, F., \& Clifton, C. (1986). The independence of syntactic processing. Journal of Memory \& Language, 25, 348-368.

FODOR, J. A. (1983). The modularity of mind. Cambridge, MA: MIT Press.

Forster, K. I. (1976). Accessing the mental lexicon. In R. Wales \& E. Walker (Eds.), New approaches to language mechanisms (pp. 257287). Amsterdam: North-Holland. 
ForSTER, K. I. (1979). Levels of processing and the structure of the language processor. In W. Cooper \& E. Walker (Eds.), Sentence processing: Psycholinguistic studies presented to Merrill Garrett. Hillsdale, NJ: Erlbaum.

Forster, K. I., \& OlbreI, I. (1973). Semantic heuristics and syntactic analysis. Cognition, 2, 319-347.

FORSTER, K. I., \& RYDER, L. (1971). Perceiving the structure and meaning of sentences. Journal of Verbal Learning \& Verbal Behavior, 10, 285-296.

Frazier, L., \& RAYNER, K. (1982). Making and correcting errors during sentence comprehenson: Eye movements in the analysis of structurally ambiguous sentences. Cognitive Psychology, 14, 178-210.

GARROD, S., \& SANFORD, A. (1977). Interpreting anaphoric relations: The integraton of semantic information while reading. Joumal of Verbal Learning \& Verbal Behavior, 16, 77-90.

Garvey, C., \& Caramazza, A. (1974). Implicit causality in verbs. Linguistic Inquiry, 5, 459-464.

Garvey, C., Caramazza, A., \& Yates, J. (1974). Factors influencing assignment of pronoun antecedents. Cognition, 3, 227-243.

Grober, E., Beardsley, W., \& Caramazza, A. (1978). A parallel function strategy in pronoun assignment. Cognition, 6, 117-133.

Hirst, W., \& BRILL, G. (1980). Contextual aspects of pronoun assignment. Journal of Verbal Learning \& Verbal Behavior, 19, 168-175.

JACKENDOFF, R. (1972). Semantic interpretation in generative grammar. Cambridge, MA: MIT Press.

MARSLEN-WILSON, W. (1973). Linguistic structure and speech shadowing at very short latencies. Nature, 244, 522-523.

MARSLEN-WILSON, W. (1975). Sentence perception as an interactive parallel process. Science, 189:4198, 226-228.

Marslen-Wilson, W., \& Tyler, L. K. (1975). Processing structure of sentence perception. Nature (London), 257, 784-786.

MARSLEN-Wilson, W., \& TYLER, L. K. (1980a). The temporal structure of spoken language understanding. Cognition, 8, 1-71

MARSLEN-Wilson, W., \& TYler, L. K. (1980b). Towards a psychological basis for a theory of anaphora. In J. Kreiman \& A. Ojeda (Eds.), Papers from the Parasession on Pronouns and Anaphora. Chicago: Chicago Linguistics Society.

Marslen-Wilson, W., Tyler, L. K., \& Seidenberg, M. (1978). Sentence processing and the clause-boundary. In W. Levelt \& G. D'Arcais (Eds.), Studies in the perception of language (pp. 219-246). New York: Wiley.

Marslen-Wilson, W., \& Welsh, A. (1976). Processing interactions and lexical access during word recognition in continuous speech. $C o g$ nitive Psychology, 10, 29-63.

Matthews, A. (1986). Pronoun resolution in two-clause sentences. Unpublished doctoral dissertation, City University of New York.

MCCAWLEY, J. (1968a). Concerning the base component of a transformational grammar. Foundations of Language, 4, 243-269.

MCCAWLEY, J. (1968b). The role of semantics in grammar. In E. Bach \& R. Harms (Eds.), Universals in linguistic theory (pp. 124-169). New York: Holt, Rinehart \& Winston.

McKoon, G., \& RATCLIF, R. (1980). The comprehension processes and memory structures involved in anaphoric reference. Journal of Verbal Learning \& Verbal Behavior, 19, 668-682.

MURPHY, G. L. (1984). Establishing and accessing referents in discourse. Memory \& Cognition, 12, 489-497.

Rayner, K., Carlson, M., \& Frazier, L. (1983). The interaction of syntax and semantics during sentence processing: Eye movements in the analysis of semantically biased sentences. Journal of Verbal Leaming \& Verbal Behavior, 22, 358-374.

TOWNSEND, D. J., \& BEVER, T. (1982). Natural units of representation interact during sentence comprehension. Joumal of Verbal Learning \& Verbal Behavior, 21, 688-703.

TyLER, L. K., \& MARSLEN-Wilson, W. (1977). The on-line effects of semantic context on syntactic processing. Joumal of Verbal Learning \& Verbal Behavior, 16, 683-692.

Tyler, L. K., \& MarsLeN-WiLSON, W. (1982). Speech comprehension processes. In J. Mehler, E. C. T. Walker, \& M. Garrett (Eds.),
Perspectives on mental representations (pp. 169-184). Hillsdale, NJ. Erlbaum.

VAN RIEMSDIJK, H., \& WILLIAMS, E. (1986). Introduction to the theory of grammar. Cambridge, MA: MIT Press.

\section{APPENDIX A \\ Materials for Experiment 1}

The VERBing NOUNs expressions for the first 28 items are drawn from the materials used by Tyler and Marslen-Wilson (1977). Each item is shown in its control version. The experimental version is derived from this form by replacing the subject of the initial subordinate clause with they.

1. As the birds soar gracefully above the field, flying kites...

2. When the men use a machine to do the work, drying clothes...

3. If the newlyweds want to take a cheap vacation, visiting relatives...

4. Since the animals seem obedient and docile, riding horses...

5. When the entertainers don't feel like putting on a show, amusing people..

6. Although the runners must rely on a dedicated coach, training athletes...

7. Since the donuts don't look very appetizing, frying eggs...

8. Even though the daisies can often become quite attractive, growing flowers...

9. Since the predators benefit from newly enacted conservation laws, hunting eagles...

10. While the boxes usually come with several internal partitions, packing cases..

11. If the engines can handle the intense competition, racing cars...

12. Since the officials presume that basic needs are not being met, starving children...

13. Although the vegetables don't take up so very much time, cooking apples..

14. Even though the captains need to rely on well-trained crews, sailing ships...

15. Because the evaluators may appear unexpectedly during the meeting, folding chairs...

16. As long as the birds stay reasonably close to the nest, breeding pigeons...

17. When the results fall into a familiar pattern, playing cards...

18. If the beginners try to thread too small a needle, shaking hands...

19. When the wheels get stuck in very deep winter snow, moving cars...

20 . Because the poems usually require careful pronunciation, rhyming words...

21. Although the fires do enormous ecological damage, burning trees...

22. Since the fragments sometimes cut people standing nearby, breaking glasses...

23. Since the noise can become extremely irritating, ringing bells...

24. Because the saws contribute most of the noise in the carpentry shop, cutting boards... 


\section{APPENDIX A (Continued)}

25. Since the runoff can provide a valuable supply of cold water, melting glaciers...

26. When the passengers arrive at better-designed airports, landing planes...

27. Since the colors affect the entire appearance of a living room, moving pictures...

28. Although the toys once brought endless amusement to children, spinning tops...

29. Supposing that the cats won't do any very serious harm, battling lions...

30. Whether the customers mean to or just can't do otherwise, hurrying people...

31. Unless the ideas amount to a definite proposal, inviting suggestions...

32. Once the coins start to make any kind of noise, jangling keys...

33. Because the clerks sometimes behave very offensively, jostling shoppers...

34. That the newborn lack teeth presents no major problem, nursing infants...

35. Except that the trainers must deal with an enormous amount of mud, bathing elephants...

36. Except that the potatoes may get very hot too quickly, boiling vegetables...

37. Although the coaches imply that few schools can maintain a long winning streak, losing games...

38. For all the fear the sounds provoke on typical occasions, clanking chains...

39. Because the freshman may overburden existing facilities, enrolling students...

40. Before the situation gets completely out of control, nagging adolescents...

\section{APPENDIX B \\ Criteria for Materials Construction}

In some cases minor divergences from these criteria were accepted where this did not seem to threaten the interpretability of the experiment.

For VERBing NOUNs expressions (i.e., the ambiguous expressions):

1. Each expression should be ambiguous with stress on the noun.

2. The stem verb in each expression should fit into both the following frames:
a. the NOUNs are VERBing
b. somebody is VERBing the NOUNs

3. Each VERBing NOUNs expression should be unique. Furthermore, there should be no repetition of any of the constituent participial forms or nouns.

For all sentence fragments:

4. Neither of the alternating verb phrases (cf. save money vs. believe that in item 1 under "Structural Cases" in Appendix C) should exhibit any associative or pragmatic relation to the VERBing NOUNs expression.

\section{APPENDIX B (Continued)}

5. On the gerundive nominal interpretation of each VERBing NOUNs expression (e.g., for John to be flying kites...), the fragment should not be odd with they taken as the implicit subject of the gerundive nominal itself.

6. For all structural cases and for the non-anomalous selection and pragmatic cases, when the VERBing NOUNs expression is taken as a plural noun phrase, it must be usable as the subject of the item whether is or are is the probe verb.

Criteria bearing on only some fragments:

7. In the structural cases, there should be no repetitions of the verbs used in the anomalous cases.

8. Except in the structural cases, no plural noun phrase should appear between the alternating verb position (e.g., the position of use and eat in item 1 under "Selection Cases" in Appendix C) and the VERBing NOUNs expression.

\section{APPENDIX C Materials for Experiment 2}

Each item in the materials listed below represents a set of four sentence fragments. Each item is shown with they as its subject. This pronoun alternated with the control form you. This alternation was crossed by an alternation between the two expressions within slashes in the verb phrase of the initial subordinate clause in each fragment. The first expression implements the non-anomalous condition and the second the anomalous condition.

\section{Structural Cases}

1. If they want to /save money/ believe that/visiting uncles...

2. Granting that they often/feel insecure/pretend that/ starving workers...

3. Though they seem to /require clarification/imply that/ demanding tests...

4. Once they begin to /consider details/accept that/ prosecuting officials..

5. So long as they sometimes /move suddenly/expect that/feeding infants...

6. Providing they intend to /avoid trouble/agree that/ pleasing associates...

7. However much they may want to /express annoyance/assume that/ pestering children..

8. Considering that they often want to /avoid scenes/proclaim that/ parting friends...

9. Supposing that they like to /frighten children/hope that/ battling lions...

10. Just because they frequently /reduce costs/require that/ boiling vegetables...

11. Granting that they like to /speak plainly/think that/ worrying mothers...

12. Even though they sometimes /work quietly/anticipate that/ investigating officers...

13. While they may /interest passersby/declare that/moving displays...

14. Now that they occasionally /live alone/insist that/admiring grandparents. 


\section{APPENDIX C (Continued)}

15. Because in some instances they may /do anything/claim that/ jostling shoppers...

16. Whenever they might/doubt something/deny that/ incriminating witnesses...

17. In that they might seem to /encourage disorder/suggest that/ developing ideas...

18. If they sometimes strive to /satisfy expectations/find that/ accommodating people...

19. Although most times they won't want to /cause trouble/propose that/ assisting coaches...

20. Supposing they sometimes/regret misbehavior/say that/ loving fathers...

21. In that they occasionally /seem uncertain/hint that/shaking hands...

22. While they often /fear losses/state that/ prodding salesmen...

23. Often when they /begin play/predict that/ deceiving opponents...

24. Except that they may /fear retaliation/discover that/ threatening strangers...

\section{Selection Cases}

1. Even though they /use/eat/ very little oil, frying eggs...

2. Just because they /make/expect/ a lot of scary noise in the dungeon, rattling chains...

3. Admitting that they rarely /disturb/observe/ the rule of quiet in the library, turning pages...

4. Whatever they may /reveal/believe/ about personal wealth, accumulating investments...

5. Except that they /need/prefer/ relatively little spice, cooking apples...

6. Providing that they /start/speak/ from reliable information, narrowing differences...

7. Because they may /encourage/dislike/ an atmosphere of genuine excitement, challenging ideas.

8. Even though they /seem/hope/ to benefit directly, growing flowers...

9. As they /sail/stroll/ in the afternoon sun, flying kites...

10. Whenever they /appear/sing/ during the procession, parading horses...

11. Whether or not they may/demonstrate/conceive/ an important concept, spinning tops...

12. When they /encourage/anticipate/ confusion among the general public, changing laws...

13. Since they /require/drink/ lots of water, melting glaciers...

14. Seeing that they /aggravate/respect/my Uncle George, ringing buzzers...

15. In that they rarely /improve/manufacture/ a high quality product, warping boards...

16. Unless they /attack/shout/ quite vigorously, overpowering animals...

17. Assuming that they don't /approach/talk/ before Harry makes his move, charging bears...

18. If they can /provide/describe/ an ample source of food, breeding pigeons...
19. Although they may /go/ache/from dawn to dusk during the harvest, working tractors...

20. While they should/detect/report/ any possible disturbance, watching dogs...

21. Even though they may /work/gossip/throughout every shift at the factory, operating machines...

22. Now that they /benefit from/disagree with/ the expansion of the conservation program, hunting eagles...

23. Considering that they /need/enjoy/ the improvements in acceleration, passing trucks...

24. While they may /irritate/like/ everybody in town, clanking bells...

\section{Pragmatic Cases}

1. Once they begin /making/suppressing/ any sort of noise, jangling keys...

2. Whenever they/smile/lecture/during the procedure, charming babies. .

3. Since they often /add/pulsate/ to the sound of the music, popping corks...

4. Because they /emphasize/analyze/ every figure of speech, rhyming phrases...

5. Supposing they will /insult/arrest/ some of the witnesses, burning witches...

6. However little they/wiggle/chirp/ when the sun comes up, waking kittens...

7. Although they may /greet/baptize/ each new member of the team, training athletes...

8. Whereas they often /replace/enclose/ the actual gold bars in some bank vault, circulating coins...

9. Sometimes when they /step/leap/ right into the water, bathing elephants...

10. Presuming that they continuously /lose/absorb/ water during the process, drying clothes...

11. As long as they /talk/complain/ all of the time, entertaining guests...

12. Considering that they / rehearse/direct/ each overture with great care, leading orchestras...

13. When they /respond/hold/ to the flight controls, landing planes...

14. Whereas they /move/grow/ rapidly in times like these, decreasing expenditures...

15. However much they may /resist/bend/ when the pressure increases, breaking glasses...

16. Given that they /jangle/calm/ most people's nerves, irritating adolescents...

17. When they /describe/grow/ long white beards, amusing boys...

18. Unless they /interest/repel/ every member of the committee, inviting suggestions...

19. Since they usually /avoid/attack/ major enemy positions, withdrawing troops...

20. Whether or not they/go/stroll/ near to the cash register, hurrying clerks...

21. Unless they /laugh/grouch/ all the time, enchanting youngsters... 


\section{APPENDIX C (Continued}

22. Assuming that they don't/shake/twist/ the shift levers out of position, racing cars...

23. Because they/consider/grade/ certain assignments very rigorously, enrolling students...
24. Unless they /accept/deny/ the existence of God, teaching nuns...

(Manuscript received February 25, 1986;

revision accepted for publication October 11, 1986.) 